Marzena ChrobaK

ORCID: 0000-0003-1386-9859

Université Jagellonne de Cracovie marzena.chrobak@uj.edu.pl

\title{
Marta Paleczna
}

ORCID: 0000-0001-7287-8734

Université Jagellonne de Cracovie marta.paleczna@doctoral.uj.edu.pl

\section{COMMUNICATION EN LANGUES ÉTRANGÈRES AVEC LES VISITEURS D'UN LIEU DE MÉMOIRE : UN SUJET PÉRIPHÉRIQUE DES ÉTUDES DE TRADUCTION}

\section{DES CENTRES ET DES CARTES}

La structuration de la réflexion en termes de centre et périphérie suppose l'existence d'une carte. Comment se présente-elle en 2020 ? Les centres traditionnels de recherche traductologique du $\mathrm{XX}^{\mathrm{e}}$ siècle - les écoles de Paris, de Leipzig, de Tel Aviv - ont perdu de leur importance. Parmi les centres de recherche contemporains, qui sont légion, comment distinguer ceux qui ont vraiment un statut central ${ }^{1}$ ? Ceci dit, ne faudrait-il pas chercher, plutôt que des écoles, des figures centrales ? Ou plutôt ne faudrait-il pas penser en catégories de centres mobiles, construits ponctuellement par les grands événements du domaine tels que le Congrès Mon-

${ }^{1}$ Il est peut-être plus facile de définir la périphéricité. La périphérie, c'est quand ce sont les autres qui écrivent ton histoire de la traduction : voici qu'une histoire de la traduction littéraire en Europe médiane - donc en Pologne, entre autres — vient de paraître en volume, conçue par les chercheurs de l'Inalco ( $c f$. A. Chalvin et al. (dir.), Histoire de la traduction littéraire en Europe médiane. Des origines à 1989, Presses Universitaires de Rennes, Rennes 2019), alors que nous Polonais n'avons toujours pas écrit notre propre version... 
dial de Traductologie initié par la Société Française de Traductologie en 2017 ? $\mathrm{Ou}$ peut-être considérer comme plus importants que les centres de recherche (de production du savoir) les centres de diffusion de ce savoir? Nous pouvons donner comme exemples les maisons d'édition comme Routledge et John Benjamins, les collections comme « Translatio » des Classiques Garnier et « Traductologie » des éditions Artois Presses Université, les revues comme Meta, la plus ancienne (1955), Babel, Target, TTR : Traduction, Terminologie, Rédaction, JoSTrans (The Journal of Specialised Translation), Palimpsestes, ou en Pologne, Między Oryginatem a Przekładem, Przekładaniec, Rocznik Przekładoznawczy... Il faudrait signaler aussi The Journal of Audiovisual Translation, première revue scientifique au monde consacrée entièrement à la Traduction Audiovisuelle, dont le numéro inaugural est sorti en 2018, lancé, entre autres, par une jeune chercheuse de l'Université Jagellonne, Anna Jankowska. C'est un nouveau-né, mais il est en anglais, en accès libre sur internet, et des auteurs réputés ont contribué au premier numéro. La revue a ainsi des chances d'occuper dans l'avenir une place centrale parmi les grandes.

Et une dernière question que nous poserons ici est la suivante : les centres universitaires sont-ils toujours les principaux agents de la recherche traductologique ? Sont-ils toujours les principaux producteurs et détenteurs du savoir dans ce domaine ? Depuis un certain temps, beaucoup se fait en dehors de l'université. Nous pensons notamment aux associations professionnelles telles que l'EST (European Society for Translation Studies) qui a d'ailleurs organisé en 2013 un colloque portant pour titre «Translation Studies: Centres and peripheries », ou à la TAUS (Translation Automation User Society), bref à des associations sans but lucratif qui produisent et diffusent le savoir sur les techniques et le marché de la traduction, sur les normes, les bonnes pratiques, les qualités et compétences requises du traducteur, qui s'engagent dans la description, la critique et la formation en traduction. En voici quelques exemples français :

- la Société Française des Traducteurs, syndicat professionnel:

É. Fusilier-Jenkinson et T. Intrieri-Mercier, Le Vade-mecum de l'expert traducteur et interprète (2016)

Une enquête métier consacrée aux interprètes (2016)

La revue Traduire;

- 1'Association des Traducteurs Littéraires de France:

Guide de la traduction littéraire (2013)

le Code des usages, le Code de déontologie du traducteur, un glossaire

le rapport La condition du traducteur (2011)

La revue TransLittérature;

- 1 'Association des Traducteurs Adaptateurs de 1'Audiovisuel:

Guide du traducteur. Formation, statuts, gestion...

Doublage, narration et voix-over, sous-titrage de $A$ à $Z$

La revue L'Écran Traduit. 
L'on en trouvera de semblables en Pologne et dans d'autres pays. Ils diffusent ce savoir sur internet, souvent en accès libre. Les chercheurs, les étudiants et les praticiens n'ont qu'à en profiter.

Centres mobiles? Centres de diffusion ? Centres en dehors de l'université ? Tous ces concepts brouillent la géographie traditionnelle de la traductologie européenne.

Mais la carte que nous proposons d'examiner plus attentivement est celle de la traductologie, Holmes' basic map of Translation Studies, dressée en 1995 par Gideon Toury à partir d'un article de James Holmes de 1988², complétée en 2009 par Andrew Chesterman ${ }^{4}$ qui y a ajouté les études portant sur le traducteur. Considérée incomplète par plusieurs chercheurs, elle le paraît surtout quand on l'applique à l'interprétologie (études en interprétation). Il nous semble qu'il faudrait y ajouter une branche : celle des études partielles restreintes dans le milieu où l'interprétation est effectuée, dans ce que Franz Pöchhacker appelle « le contexte social de l'interaction, ou le cadre dans lequel l'interprétation est exécutée » (trad. M.C. $)^{5}$ (figure 1$)^{6}$.

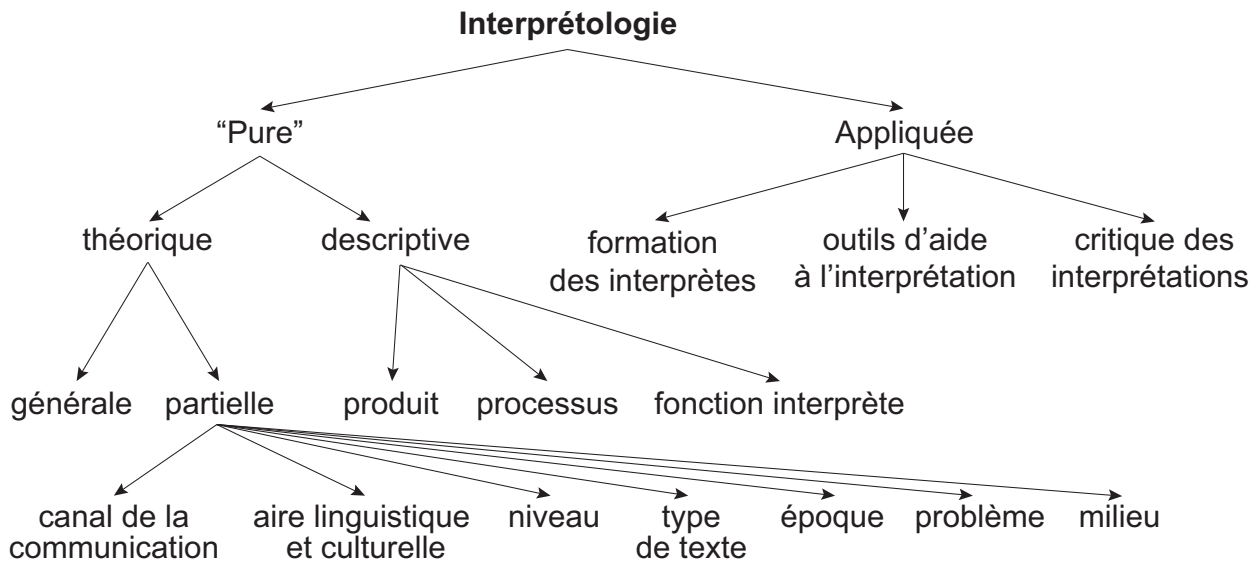

Figure 1. Proposition de carte de l'interprétologie

${ }^{2}$ G. Toury, Descriptive Translation Studies and Beyond, John Benjamins, AmsterdamPhiladelphia 1995.

3 J. Holmes, The Name and Nature of Translation Studies, Amsterdam 1988.

4 A. Chesterman, « The Name and Nature of Translator Studies », Hermes 42, 2009.

${ }^{5}$ F. Pöchhacker, « The social context of interaction, or setting where interpreting is carried out », [dans :] idem, Introducing Interpreting Studies, Routledge, London-New York 2004, p. 13.

${ }^{6}$ Une carte conçue entièrement dans la perspective des études en interprétation, avec la personne de l'interprète comme objet central, vient d'être proposée par un chercheur polonais : M. Walczyński, Psycho-Affective Factors in Consecutive Interpreting, Peter Lang, Berlin 2019, p. 74. 
Or, l'examen des publications relatives à cette branche ${ }^{7}$ révèle que c'est l'interprétation de conférence, surtout pour de grandes institutions internationales, qui se trouve en position centrale, au centre de l'intérêt des chercheurs. Ensuite, vient l'interprétation au tribunal, au poste de police, dans le contexte des demandes d'asile, pour les services de santé, pour la sphère diplomatique, à la guerre et en zone de conflit, pour les médias, dans le milieu religieux, dans le milieu sportif, tandis que celle pratiquée dans le monde des affaires, les milieux scolaires, académiques et culturels, le tourisme, le domaine privé ou familial reste parfaitement en périphérie de la recherche ${ }^{8}$.

La situation est identique en Pologne. Les études portant sur l'interprétation autre que de conférence, pour le tribunal et les services publics ou en zone de conflit sont rares, ponctuelles et dispersées ${ }^{9}$. Il n'existe, à notre connaissance, aucune étude traitant de l'interprétation en milieu privé ou familial ou pour les touristes.

C'est de la constatation de cette lacune qu'est née l'idée d'une thèse de doctorat intitulée Tłumaczenie wyjątkowej lekcji. Przektad ustny dla obcokrajowców zwiedzajacych Państwowe Muzeum Auschwitz-Birkenau (PMAB) [Traduire une leçon exceptionnelle. Interprétation en langues étrangères pour les visiteurs du Musée d'État d'Auschwitz-Birkenau], rédigée en polonais à partir de 2017 par Marta Paleczna, diplômée de philologie espagnole de la faculté de philologie de l'Université

7 Cf. F. Pöchhacker, op. cit. ; entrées consacrées à l'interprétation dans : C. Millán, F. Bartrina, Routledge Handbook of Translation Studies, Routledge, London-New York 2013 ; tables des matières de revues telles que Interpreting. International Journal of Research and Practice in Interpreting ; K. Takeda, J. Baigorri-Jalón, New Insights in the History of Interpreting, John Benjamins, Amsterdam 2016 ; D. Gile, Traditions and Innovation in Interpreting Studies. A Personal Analysis for 2016, t. 11(5), Domínios de Lingu@gem, Uberlândia 2017, pp. 1424-1439.

8 Dans un entretien personnel, pendant le colloque «Points of View in Translation and Interpreting », le 23 juin 2017, à Cracovie, Pöchhacker a été d'accord avec nous.

9 Citons à titre d'exemple : M. Dąbrowski, J. Redlich, M. Stanisz, « "Interprète naturel” dans le milieu étudiant. Sur l'exemple des étudiants du programme Erasmus », [dans :] E. Skibińska, R. Solová, K. Gostkowska (dir.), Vingt-cinq ans après... Traduire dans une Europe en reconfiguration, Orizons, Paris 2015 ; M. Chrobak, Między światami. Ttumacz ustny oraz komunikacja międzykulturowa w literaturze odkrycia i konkwisty Ameryki, Wydawnictwo Uniwersytetu Jagiellońskiego, Kraków 2012, chapitres : " Miłość do pieniądza jest poliglotą », "Thumacze pielgrzymów »; O. Furmanek, S.M. Bałdyga OFM, "Faith-related interpreting. Simultaneous interpreters" team at World Youth Day, Kraków 2016 », [dans :] B. Marczuk, I. Piechnik (dir.), Discours religieux : langages, textes, traductions, Biblioteka Jagiellońska, Kraków 2020, pp. 131-147 ; K. Gałązka, « Tłumacz medyczny w Polsce », [dans :] M. Tryuk (dir.), O tlumaczach, prawnikach, lekarzach i urzędnikach, Wydział Lingwistyki Stosowanej Uniwersytetu Warszawskiego, Warszawa 2010, pp. 111-155; L. Harmon, « O nietypowych rolach thumacza nieformalnych spotkań biznesowych », Między Oryginatem a Przektadem, R. XXIV, $\mathrm{n}^{\circ}$ 2(40), 2018, pp. 123-137 ; L. Wille, « Interpreting Informal Business Meetings. Pitfalls Beyond Language », [dans :] M. Grygiel, M. Rzecka (dir.), Specialist Communication in Education, Translation and Linguistics, Wydawnictwo Uniwersytetu Rzeszowskiego, Rzeszów 2017, pp. 195-206. 
Jagellonne de Cracovie (Pologne). Voici les premiers résultats de la recherche empirique observationnelle (naturaliste) qu'elle a menée dans le cadre de cette thèse.

\section{INTERPRÉTER POUR LES VISITEURS DU MUSÉE D’ÉTAT D'AUSCHWITZ-BIRKENAU}

Le Musée d'Auschwitz-Birkenau a été créé en 1947 sur le site du plus grand complexe concentrationnaire nazi, à la fois camp de concentration et camp d'extermination. En raison de sa taille, Auschwitz est considéré comme le symbole des meurtres de masse commis par les nazis, et plus particulièrement de la Shoah, au cours de laquelle près de six millions de Juifs furent assassinés. Monument historique et culturel majeur du « devoir de mémoire », le Musée d'Auschwitz-Birkenau est depuis 1979 inscrit au patrimoine mondial de l'Unesco.

Le musée est visité actuellement par environ deux millions de personnes par an ${ }^{10}$, ce qui en fait l'un des sites les plus fréquentés de Pologne ${ }^{11}$. La majorité des visiteurs sont polonais, mais le nombre des étrangers augmente constamment. En 2018, ils venaient surtout du Royaume Uni (281 700), des États-Unis (136 400), d'Italie (116 900), d'Espagne (95 100), d'Allemagne (76 300) et de France $(69000)^{12}$.

La visite du musée est individuelle ou guidée. Les guides officiels ${ }^{13}$, appelés « éducateurs ", s'expriment soit en polonais, soit dans une des langues étrangères suivantes : allemand, anglais, croate, coréen, espagnol, français, hébreu, hongrois, italien, japonais, néerlandais, norvégien, russe, roumain, serbe, slovaque, suédois, tchèque ${ }^{14}$. Les guides parlant des langues étrangères sont environ $300^{15}$. Ce nombre n'est cependant pas suffisant, et lorsqu'il est impossible de réserver une visite guidée dans une langue étrangère, les groupes organisés de visiteurs en réservent une en polonais et viennent avec un interprète qui traduit les explications du guide pendant la visite ; souvent, c'est l'accompagnateur polonais du groupe qui assure l'interprétation. Les groupes asiatiques réservent souvent la visite guidée en anglais et viennent avec leur propre accompagnateur-interprète.

10 B. Bartyzel, P. Sawicki (dir.), Sprawozdanie 2018, Państwowe Muzeum Auschwitz-Birkenau w Oświęcimiu, 2019, p. 23.

11 Z. Bańka et al. (dir.), Kultura w 2018, Zakład Wydawnictw Statystycznych, WarszawaKraków 2019,p. 92, disponible sur: <https://stat.gov.pl/obszary-tematyczne/kultura-turystyka-sport/ kultura $>$ [consulté le 11.01.2020].

12 B. Bartyzel, P. Sawicki, op. cit., p. 25.

13 Diplomés de l'enseignement supérieur (principalement en histoire, philologie, sciences de l'éducation), formés par le Musée d'Auschwitz-Birkenau.

$14<$ http://auschwitz.org/zwiedzanie/przewodnicy/> [consulté le 5.01.2020].

15 B. Bartyzel, P. Sawicki, op. cit., p. 22. 
Marta Paleczna a mené deux enquêtes auprès des interprètes. La première a été organisée en septembre 2017 auprès de 10 interprètes dont la langue de travail était l'espagnol et qui ont répondu à un questionnaire de 6 questions ouvertes $^{16}$. La deuxième s'est déroulée de juin 2018 à septembre 2018 et a été réalisée au moyen de SURVIO, un système d'enquête en ligne. Trente-trois interprètes travaillant avec des langues différentes ont complété un questionnaire contenant cette fois une trentaine de questions ${ }^{17}$. Les deux enquêtes portaient principalement sur les problèmes auxquels les interprètes sont confrontés dans leur travail.

Nous nous attendions à ce qu'une des premières difficultés majeures soit la traduction des mots-témoins, des références à la réalité du camp de concentration et d'extermination, des mots et expressions renvoyant à des objets, des personnages, des activités, des lieux de l'univers inconcevable et cauchemardesque qu'était le camp de concentration et d'extermination, ainsi que la traduction des mots de l'argot du camp, la Lagersprache, lingua franca des détenus, à la grammaire simplifiée et au lexique comprenant des mots allemands, des mots allemands polonisés, des dialectalismes polonais et allemands, des sigles et abréviations polonaises et allemandes, des néologismes polonais, des mots yiddish et, au fur et à mesure qu'affluaient les déportés des autres pays d'Europe, des emprunts à d'autres langues telles que le slovaque, le hongrois, le français, l'italien ou le grec.

Les interprètes ont donc été invités à donner des exemples de tels mots-témoins et à expliquer s'ils posaient des difficultés en traduction. Ils ont donné des exemples de mots qu'ils considéraient comme difficiles : "Noms des fonctions des prisonniers (chef de bloc, chef de pièce, etc.), expressions appartenant au jargon du camp $»^{18}$, mais en même temps, à notre surprise, presque tous les répondants (29 sur 33) ont déclaré que la difficulté surgissait uniquement au début de leur travail d'interprète, c'est-à-dire à la première traduction ou pendant les préparatifs de la première traduction, et qu'elle disparaissait ensuite :

C'est juste une question de préparation initiale/de vérification de plusieurs dizaines de mots spécialisés. [Ce n'est] pas [un problème] si le traducteur se prépare.

C'est un GROS problème au tout début, parce que ce ne sont pas des mots qui sont utilisés dans des conversations normales, mais après un certain temps, on se les rappelle $[\ldots]^{19}$.

16 Pour la discussion des résultats de cette enquête, $c f$. M. Paleczna, « Przekład ustny dla obcokrajowców w Państwowym Muzeum Auschwitz-Birkenau (PMAB): warunki pracy, problemy i profil thumacza », Politeja 3(60), 2019, pp. 167-182.

17 Cf. Annexe 1.

18 « Nazwy funkcji więźniów (blokowy, sztubowy etc.), określenia należące do żargonu obozowego ». La traduction de toutes les citations des réponses au questionnaire est de M.C.

19 « Jest to kwestia wstępnego opracowania/sprawdzenia kilkudziesięciu specjalistycznych słów. Nie, jeśli tłumacz się przygotuje. DUŻY problem sprawia na samym początku, bo nie są to słowa, których używa się w normalnych rozmowach, natomiast po chwili już się je pamięta [...] ». 
En effet, nous aurions dû nous en douter, vu la répétitivité relative — mais non absolue — de la visite guidée :

Chacun a sa propre narration, mais les faits de base se répètent.

Les informations de base, bien sûr, se répètent, mais chaque éducateur a sa propre façon de guider et sa propre façon de raconter le récit. Il existe donc également des différences assez importantes. Il existe également certaines questions litigieuses sur lesquelles les éducateurs ne sont pas d'accord.

Généralement, ils se répètent - la base reste la même. Ils diffèrent tout au plus par des récits supplémentaires adaptés aux besoins, aux types et aux attentes du groupe ${ }^{20}$.

$\mathrm{Vu}$ le grand nombre de publications traitant du sujet en langues étrangères, dont certaines sont disponibles à la boutique du musée, il existe une sorte de base lexicale « officielle » des réalités du camp dans les différentes langues. Mais un doute persistait malgré tout : même si les interprètes savent comment traduire ce type de mots, même s'ils connaissent leurs équivalents dans la langue cible, ces mots inhabituels sont-ils compris par leurs destinataires ? Marta Paleczna a posé la question à des visiteurs.

En effet, l'un des points forts de cette recherche est de vouloir connaître l'avis de toutes les trois parties impliquées dans cette interaction qu'est la visite de musée interprétée : pas seulement l'avis de l'interprète, mais aussi celui de l'émetteur du message, c'est-à-dire le guide/éducateur, et de son récepteur, c'est-à-dire le visiteur.

Marta Paleczna a obtenu environ 100 réponses à un bref questionnaire ${ }^{21}$ distribué par ses collègues accompagnateurs aux visiteurs, principalement de langue espagnole, pendant le trajet de retour du musée à Cracovie, à l'été 2019. Presque tous ont déclaré ne pas avoir eu de problèmes de compréhension des références concentrationnaires ; les seuls à signaler des difficultés de compréhension ont été quelques visiteurs qui ont suivi la visite en anglais sans être des locuteurs natifs de cette langue.

L'une des raisons de cette bonne compréhension pourrait être le caractère largement audiovisuel de la traduction au musée, où un nombre important d'informations sont transmises par des canaux non-verbaux, notamment par l'image : les édifices, les objets exposés, les maquettes, les photos. Le discours et l'image s'expliquent mutuellement. Par exemple, on peut ne pas comprendre tout de suite le mot polonais pasiak, uniforme rayé (blouse, pantalon, calot) porté obligatoirement par tous les détenus, mais quand on le voit sur toutes les photos des déportés et exposé dans les vitrines, on comprend le sens du mot. Les interprètes s'en rendent compte, eux aussi :

20 « Każdy ma swoją opowieść, ale zasadnicze fakty się powtarzają. Podstawowe informacje oczywiście się powtarzają, jednak każdy edukator ma swój sposób oprowadzania i własną wypracowaną narrację, więc zdarzają się też dość duże różnice. Istnieją też pewne kwestie sporne, co do których poszczególni edukatorzy nie są zgodni. W większości się powtarzają — część bazowa jest taka sama. Różnią się co najwyżej w dodatkowych historiach dopasowanych do potrzeb, rodzaju i oczekiwań grupy ».

${ }^{21} C f$. Annexe 2. 
[Ce qui facilite notre travail, c'est la] possibilité de voir ce dont parle le guide; [Ce qui facilite notre travail, c'est] le fait que les visiteurs voient de quoi on parle, donc même s'il nous manque un mot, nous pouvons utiliser ce qu'on voit ${ }^{22}$.

Certains répondants (11 sur 33) ont signalé aussi des difficultés relatives à la traduction du lexique de spécialité : vocabulaire chimique, médical, technique ; cela est certainement dû à la formation non-technique, majoritairement philologique (16 sur 33), des interprètes. Une personne a signalé le phénomène de détournement sémantique de certains mots, détournement visible déjà dans la dénomination du Konzentrationslager, camp de concentration, qui était en fait un camp d'extermination : « Le terme même de "camp de concentration" pose problème car il ne reflète pas la vérité : il ne s'agissait pas de concentration, au Konzentrationslager Auschwitz » ${ }^{23}$.

Si ce n'est pas le lexique spécialisé, quelle serait donc la difficulté majeure du travail des interprètes ? C'est le manque de temps, la contrainte temporelle. La visite standard dure 3 heures et demie et il est impossible de la prolonger à cause du grand nombre de visiteurs. En 2016, ce nombre a dépassé les deux millions par an. Cela donne en moyenne plus de 5500 visiteurs par jour, ou 600 environ par heure, parfois dispersés sur les 170 hectares du camp, mais souvent entassés dans l'espace restreint d'un baraquement ou d'une salle d'exposition. La durée de la visite est la même pour tous les groupes, avec ou sans interprète, il faut donc aller vite - se déplacer vite, parler vite, interpréter vite, écouter vite, tout en respectant le caractère exceptionnel de cet endroit, et en permettant aux visiteurs de poser des questions, de réfléchir, de se recueillir. Les interprètes signalent que certains guides ne voient pas de différence entre une visite guidée avec ou sans interprétation :

Certaines personnes comprennent la coopération avec le traducteur, mais d'autres éducateurs délivrent leurs informations de la même manière que pour un groupe sans interprète ; [vous posez une] très bonne question : [les guides donnent] certainement trop de détails. Ils ne comprennent pas que la traduction double le temps de la visite ${ }^{24}$.

Quelles stratégies ou techniques développent donc les interprètes ? Les mêmes que celles employées dans l'interprétation simultanée :

1) Réduction au minimum du décalage entre l'écoute du message source et la production du message cible.

22 « [Ułatwia nam pracę] możliwość zobaczenia tego, o czym mówi przewodnik; [Ułatwia nam pracę] fakt, że zwiedzający widzą, o czym się mówi, więc nawet jeśli brakuje jakiegoś słowa, można się posiłkować tym, co widać ».

23 «Problematyczne jest samo sformułowanie "obóz koncentracyjny", ponieważ nie oddaje tego, co tam było, nie o koncentrację chodziło w KL Auschwitz ».

24 « Część osób rozumie współpracę z tłumaczem, część edukatorów przekazuje informacje w sposób identyczny jak grupie, która nie ma tłumacza. Bardzo dobre pytanie: zdecydowanie zbyt dużo szczegółów. Nie rozumieją, że thumaczenie to czasowo 100 plus drugie tyle ». 
2) Réduction du message source par sélection des informations à transmettre et compression de l'information.

3) Suppression du message source. En effet, il arrive souvent que l'interprète remplace le guide : 29 sur 33 déclarent avoir eu recours à ce procédé, dont 11 après 2 ou 3 visites, 4 après 10 visites ou plus.

Souvent, quand les éducateurs connaissent l'interprète, ils se fient entièrement à ses connaissances. Si le traducteur déclare connaître le sujet, l'éducateur ne fait que rappeler des chiffres ou des dates et s'assure que le traducteur a fourni les informations les plus importantes. Si le traducteur ne connaît pas le sujet, l'éducateur raconte l'ensemble du scénario de la visite, avec des phrases complètes ${ }^{25}$.

La confiance des éducateurs (guides) envers les interprètes s'explique aussi par le fait que nombre d'entre eux ont commencé leur travail au musée en qualité d'interprètes pour ensuite se former en tant qu'éducateurs. Même s'il est remplacé par l'interprète, l'éducateur reste à sa disposition et intervient pour dissiper les doutes de l'interprète ou répondre aux questions des visiteurs. Ce qui semble quand même inquiétant, c'est qu'une dizaine de répondants se considèrent prêts à remplacer l'éducateur malgré une expérience relativement courte.

4) Élargissement du temps des explications en dehors de la visite. C'est un procédé pratiqué régulièrement par les interprètes qui sont en même temps accompagnateurs des groupes organisés se rendant au musée en autocar à partir d'autres villes de Pologne, souvent Cracovie. Pendant le trajet aller, l'accompagnateurinterprète introduit les sujets qui seront abordés pendant la visite du camp, puis les complète au retour.

On pourrait se demander si la traduction simultanée ne serait pas envisageable dans le cadre d'une visite au musée. Nous avons recueilli le témoignage d'une professeure de langue interprétant occasionnellement au musée d'Auschwitz-Birkenau, qui, impressionnée par le contenu qu'elle devait transmettre, a eu recours à la simultanée, pour, dit-elle, « se mettre en mode automatique », mettre en veille son imagination, son émotion et ses autres sentiments, et se convertir ainsi en une simple machine à traduire. Cette personne était une linguiste experte, une interprète expérimentée. La plupart des accompagnateurs-interprètes, en revanche, n'ont pas de formation solide en interprétation. Or, en simultanée, l'écoute et l'analyse du message source se déroulant en même temps que sa restitution en langue cible, la production du message cible demanderait probablement une concentration qu'il est impossible d'atteindre dans les conditions d'une visite au musée, avec le bruit et le mouvement ambiants. L'effort requis dépasserait les capacités mentales et physiques d'un interprète moyen. Un répondant a commenté ainsi

25 « [Edukatorzy] Często gdy znają tłumacza, zdają się całkowicie na jego wiedzę. Jeżeli tłumacz deklaruje znajomość tematu, to edukator zazwyczaj tylko przypomina dane liczbowe albo daty i upewnia się, czy tłumacz przekazał najważniejsze informacje. Jeżeli tłumacz nie zna tematu, wtedy edukator mówi cały scenariusz zwiedzania, całymi zdaniami ». 
cette possibilité : «Les casques audio permettent d'épargner votre voix et de traduire simultanément, si on sait le faire $»^{26}$.

\section{CONCLUSION}

Le nombre de répondants de ces enquêtes n'était pas très élevé : une centaine de visiteurs, ce qui est beaucoup, mais seulement 33 interprètes ${ }^{27}$ et 6 guides. Les échantillons ne sont pas représentatifs. C'est du reste un problème typique de ce genre d'étude, signalé souvent par les chercheurs ${ }^{28}$. En effet, les professionnels sont peu enclins à consacrer leur temps à remplir des questionnaires, et encore moins à fournir des informations sur leurs pratiques professionnelles, à propos desquelles ils préfèrent rester discrets. Le nombre de répondants nous paraît donc acceptable. La difficulté de collecter des données naturelles est d'ailleurs, nous semble-t-il, l'une des raisons — sinon la raison principale — du manque d'études consacrées à ce type d'interprétation.

La recherche montre que la traduction du lexique spécialisé n'est problématique qu'au début de la pratique traductive, que le caractère audiovisuel de la visite facilite la compréhension des mots inhabituels, que le problème principal est le manque de temps et que les interprètes essaient de le pallier en réduisant et parfois en supprimant le message source. Ces réponses ne surprennent pas ceux qui connaissent la réalité de l'interprétation pour les touristes. Elles ont le mérite d'éclairer un terrain jusque là inexploré par les traductologues. En outre, cette recherche présente une description pertinente du travail des interprètes dans un musée-lieu de mémoire, vu par les trois parties impliquées dans l'interaction. Elle sera suivie par des entretiens plus pointus et plus poussés, qui permettront peutêtre d'approfondir certains points, d'éclaircir certains aspects de ce type d'interprétation.

Avec cet article, nous espérons donner l'impulsion à des recherches dans les domaines qui restent aujourd'hui en périphérie de l'interprétologie, bien qu'ils occupent une place importante dans le volume global de messages interprétés. potrafi $»$.

26 «Słuchawki pozwalają oszczędzać głos, a także tłumaczyć symultanicznie, jeśli ktoś du musée.

27 Le nombre total des interprètes est difficile à établir, il n'apparait pas dans les statistiques

28 Cf. D.A. Dillman, Mail and Internet Surveys. The Tailored Design Method, John Wiley \& Sons, New York 2000 ; M. Liu, « Methodology in interpreting studies. A methodological review of evidence-based research », [dans :] B. Nicodemus, L. Swabey, Advances in Interpreting Research, John Benjamins Publishing Company, Amsterdam-Philadelphia 2011. 


\section{ANNEXE 1 \\ QUESTIONNAIRE DESTINÉ AUX INTERPRÈTES \\ (traduit du polonais par M.C.)}

Quel est votre âge, formation, expérience du métier ? Quels sont les éléments caractéristiques de la réalité des camps de concentration et d'extermination? Le lexique du camp, pose-t-il des problèmes pendant l'interprétation ? Si oui, donnez quelques exemples. Le lexique de spécialité pose-t-il des problèmes pendant l'interprétation? Si oui, donnez des exemples des domaines de spécialité et des mots problématiques. Le lexique relatif à l'histoire et à la culture polonaises pose-t-il des problèmes pendant l'interprétation ? Si oui, donnez quelques exemples. Quels facteurs rendent l'interprétation au Musée d'Auschwitz-Birkenau difficile ? Quels facteurs la rendent plus facile ? Comment les guides transmettent-ils le contenu de la visite ? Quel type d'informations les guides fournissent-ils ? À quelle vitesse les fournissent-ils ? Le contenu fourni diffère-t-il en fonction du guide ? Les guides interrompent-ils ou commentent-ils votre interprétation ? Comment vous préparez-vous à l'interprétation ? Quelles sont les émotions qui vous accompagnent pendant l'interprétation? Quel style de transmission des informations préférez-vous (par exemple émotionnel, imagé, discret) ? Vous est-il arrivé de remplacer le guide ? Si c'est vous qui guidez, que fait le guide ? L'interprétation dépend-elle des visiteurs ? Pour quels visiteurs est-il facile d'interpréter, et pour quels visiteurs est-il difficile d'interpréter? Les visiteurs posentils beaucoup de questions pendant ou après la visite ? Quels sont les sujets qui reviennent le plus souvent dans leurs questions ? Avez-vous eu l'occasion d'accompagner un groupe de visiteurs avant ou après la visite ? Si oui, cela vous a-t-il donné l'occasion de transmettre ou de recevoir des commentaires supplémentaires concernant la visite ? Vous est-il arrivé qu'un groupe de visiteurs manifeste son mécontentement à l'idée de prendre part à une visite interprétée ?

\section{ANNEXE 2}

\section{QUESTIONNAIRE DESTINÉS AUX VISITEURS}

(traduit de l'espagnol par M.C.)

Les explications fournies par l'interprète étaient-elles claires ? Y avait-il des mots ou des expressions qui devraient être expliqués d'une façon plus détaillée ? Les mots relatifs à la vie au camp et à la réalité du camp étaient-ils suffisamment expliqués ? Le rythme auquel l'information était délivrée par l'interprète était-il adéquat? Quels sujets mentionnés (ou non) pendant la visite mériteraient d'aprèsvous d'être développés davantage? Quelles émotions accompagnent la visite? Que pensez-vous de la visite ? Comment l'évaluez-vous ? 


\title{
COMMUNICATION IN FOREIGN LANGUAGES \\ WITH THE VISITORS OF A PLACE OF MEMORY: A PERIPHERICAL TOPIC IN INTERPRETATION STUDIES
}

\begin{abstract}
After some general remarks on a contemporary basic map of Translation Studies, we present the results of a research on a peripherical topic in the field Interpretation Studies: interpreting in a museum setting. The museum concerned is the Auschwitz-Birkenau State Museum, a former nazi concentration and extermination camp situated in Poland, a World Heritage Site, and a symbol of the Holocaust. The research is based on surveys conducted in 2017 and 2018 by Marta Paleczna among the camp's visitors, guides, and interpreters. We discuss the interpreters' main problems, which include translating camp-related and other specific terms, collaboration with a guide, the increasing number of visitors and time constraint, and their solutions, which include compressing the explanations given by a guide during the visit, taking over the role of a guide by the interpreter, and lengthening the explanation time by taking advantage of the trip to the museum and back.
\end{abstract}

Key words: interpreting in a museum setting, interpreting in the Auschwitz-Birkenau State Museum, time constraint in interpreting, surveys in interpreting studies. 